\title{
Conflitos entre as equipes de saúde na transferência do cuidado pré-hospitalar
}

\author{
Conflicts between health teams in transfer of prehospital care \\ Conflictos entre equipos de salud en el traslado del cuidado prehospitalário
}

\author{
Natasha da Silva Indruczaki'; Jeanini Dalcol Miorin"; Volmir Figueiredo Pais"I'; \\ Mariana Pereira Gemelliv"; Maria Alice Dias da Silva Limav' Daiane Dal Paill
}

\begin{abstract}
RESUMO
Objetivo: compreender as causas dos conflitos entre as equipes de saúde na transferência do cuidado pré-hospitalar sob a perspectiva dos profissionais. Método: estudo qualitativo, descritivo e exploratório, realizado no Serviço de Atendimento Móvel de Urgência (SAMU) de Porto Alegre/RS. Foram entrevistados 28 profissionais do SAMU e observados 135 horas de atendimentos. Empregou-se a análise de conteúdo temática. Resultados: As causas atribuídas aos conflitos foram: (1) a superlotação dos hospitais e a pouca receptividade da equipe: os profissionais do SAMU se sentem culpabilizados pela superlotação dos serviços e punidos por meio da retenção de macas; e (2) a regulação e as (in)definições de fluxos na rede: há lacunas nas pactuações sobre o destino dos pacientes, recaindo aos profissionais o desafio de dar sequencia ao atendimento. Conclusão: o excesso de demandas e a baixa articulação dos serviços na rede causam conflitos entre as equipes de saúde na transferência do cuidado pré-hospitalar.

Descritores: Cuidado Transicional; Transferência da Responsabilidade pelo Paciente; Continuidade da Assistência ao Paciente; Serviços Médicos de Emergência.
\end{abstract}

\begin{abstract}
Objective: to understand, from the health personnel's perspective, the causes of conflicts between health care teams during transfer from pre-hospital care. Method: this exploratory, qualitative, descriptive study was conducted at the ambulance service (Serviço de Atendimento Móvel de Urgência, SAMU) of Porto Alegre, Rio Grande do Sul, Brazil. Twenty-eight SAMU personnel were interviewed and 135 hours of care service observed. Thematic content analysis was used. Results: the causes attributed to conflicts were: (1) hospital overcrowding and poor staff receptivity: SAMU personnel felt blamed for service overcrowding and punished by gurneys being withheld, and (2) the bed regulation center and (in)definition in patient flows: there were gaps in patient routing agreements, it then falling to SAMU personnel to meet the challenge of completing the service. Conclusion: excessive demands and poor coordination between services in the health care network cause conflicts between teams in transfer from pre-hospital care.
\end{abstract}

Descriptors: Transitional Care; Patient Handoff; Continuity of Patient Care; Emergency Medical Services.

\section{RESUMEN}

Objetivo: comprender, desde la perspectiva del personal de salud, las causas de los conflictos entre los equipos de salud durante el traslado desde el cuidado prehospitalario. Método: este estudio exploratorio, cualitativo y descriptivo se realizó en el servicio de ambulancia (Serviço de Atendimento Móvel de Urgência, SAMU) de Porto Alegre, Rio Grande do Sul, Brasil. Se entrevistó a 28 miembros del SAMU y se observaron 135 horas de servicio de atención. Se utilizó análisis de contenido temático. Resultados: las causas atribuidas a los conflictos fueron: (1) hacinamiento hospitalario y poca receptividad del personal: el personal del SAMU se sintió culpable por el hacinamiento del servicio y castigado con camillas retenidas, y (2) centro de regulación de camas y (in) definición en los flujos de pacientes: Hubo lagunas en los acuerdos de enrutamiento de pacientes, y luego le correspondió al personal de SAMU enfrentar el desafío de completar el servicio. Conclusion: las demandas excesivas y la mala coordinación entre los servicios de la red sanitaria provocan conflictos entre los equipos en el traslado desde el cuidado prehospitalario. Descriptores: Cuidado de Transición; Pase de Guardia; Continuidad de La Atención al Paciente; Servicios Médicos de Urgencia.

\section{INTRODUÇÃO}

O Serviço de Atendimento Móvel de Urgência (SAMU) representa um componente imprescindível na conformação da Rede de Atenção às Urgências (RAU) devido ao seu impacto significativo sobre a morbimortalidade da população, especialmente por causas externas e cardiovasculares. Ele foi instituído com o objetivo de organizar os fluxos e articular os demais serviços da rede de saúde, configurando-se um facilitador para melhoria do acesso aos serviços de urgência e aumentando a sobrevida dos usuários ${ }^{1}$. No entanto, para cumprir com seu propósito de articulação e dar continuidade a assistência do paciente, a interação do SAMU com outros serviços e equipes é fundamental para a transição do cuidado.

'Enfermeira. Residente. Programa de Atenção ao Paciente Crítico da Residência Multiprofissional em Saúde do Grupo Hospitalar Conceição. Porto Alegre. Rio Grande do Sul. Brasil. E-mail: natashasindruczaki@gmail.com. ORCID: https://orcid.org/0000-0002-2244-5428

"Enfermeira. Mestre. Doutoranda do Programa de Pós-Graduação em Enfermagem da Universidade Federal de Santa Maria. Rio Grande do Sul. Brasil. E-mail: jeaninidmiorin@hotmail.com. ORCID: https://orcid.org/0000-0001-7672-7191

I'Enfermeiro. Técnico de enfermagem. Serviço de Traumatologia do Hospital Cristo Redentor. Porto Alegre. Rio Grande do Sul. Brasil. E-mail: mirovolmir@gmail.com. ORCID: https://orcid.org/0000-0002-2834-3350

IVAcadêmica de Enfermagem. Universidade Federal do Rio Grande do Sul. Porto Alegre. Brasil. E-mail: marianagemelli@yahoo.com.br. ORCID: https://orcid.org/0000-0001-5309-5072 VEnfermeira. Doutora. Professora adjunta da Universidade Federal do Rio Grande do Sul. Porto Alegre. Brasil. E-mail: malice@enf.ufrgs.br. ORCID: https://orcid.org/0000-0002-3490-7335 v'Enfermeira. Doutora. Professora adjunta da Universidade Federal do Rio Grande do Sul. Porto Alegre. Brasil. E-mail: daiane.dalpai@gmail.com. ORCID: https://orcid.org/0000-0002-6761-0415 
A transição do cuidado é definida como um conjunto de ações destinadas a assegurar a coordenação e a continuidade das ações na transferência de pacientes entre diferentes serviços de saúde ${ }^{2}$. Assim, a transferência do cuidado é um componente da transição e envolve um intercâmbio de informações verbais e documentadas, onde é possível referenciar um paciente de um profissional de saúde para o outro, seja durante a admissão, no decorrer da assistência e na alta hospitalar ${ }^{3-4}$. Considerando a potencialidade de danos devido às inconformidades relacionadas à transferência do cuidado ${ }^{5}$, protocolos e definições de fluxos das informações são indicados ${ }^{6}$.

Além do conteúdo das informações, cabe destacar a interferência das relações interpessoais implicadas na transferência do cuidado. Por vezes, a ocorrência de conflitos entre as equipes são geradoras de tensões entre os profissionais, desgaste e fadiga, impactando sobre a segurança dos pacientes no momento da transferência do cuidado ${ }^{7}$.

$\mathrm{Na}$ atenção pré-hospitalar às urgências a transferência do cuidado tem sido preocupação expressa por órgãos de acreditação internacional ${ }^{8}$, bem como iniciativas nacionais em prol da segurança do paciente ${ }^{9}$. Transferências efetivas são maneiras de superar a fragmentação da atenção, de garantir continuidade dos cuidados e de efetivar sistemas integrados de saúde e o reflexo de uma RAU pouco articulada, com deficiências nos processos de continuidade traz implicações para a efetividade da continuidade do cuidado seguro ${ }^{10}$.

Também consta na literatura internacional que as boas relações entre as equipes funcionam como facilitadores do processo de comunicação no atendimento às urgências ${ }^{7}$, pondo em relevo os efeitos que os conflitos nas relações interpessoais podem trazer para a assistência, uma vez que os prejuízos na comunicação durante a transferência do cuidado podem levar a atrasos desnecessários no diagnóstico e tratamento, bem como eventos adversos que resultam em morte ou lesões graves aos pacientes ${ }^{4}$. Assim, compreender as causas dos conflitos entre as equipes na transferência do cuidado pré-hospitalar pode representar subsídio a investimentos nos processos relacionados às interações entre as equipes de saúde pré e intra-hospitalar.

Considerando o exposto, este estudo tem como questão norteadora: Na perspectiva dos profissionais do SAMU, qual(quais) a(s) causa(s) dos conflitos entre as equipes de saúde na transferência do cuidado pré-hospitalar? Nesse sentido, esta pesquisa teve como objetivo compreender as causas dos conflitos que ocorrem entre as equipes de saúde na transferência do cuidado pré-hospitalar na perspectiva dos profissionais.

\section{MÉTOdo}

Tratou-se de um estudo de abordagem qualitativa, do tipo descritivo-exploratório, utilizando as técnicas de observação não participante e entrevista semiestruturada.

As observações foram realizadas acerca dos atendimentos, desde a chamada enviada pelo regulador da central às equipes, as informações no local da ocorrência e as informações documentadas na ficha de atendimento, às interações do SAMU com os serviços que recebem o paciente. As observações totalizaram 135 horas e as situações relacionadas ao objeto de investigação foram registradas em diário de campo.

As entrevistas ocorreram nas bases do SAMU e foram conduzidas por meio de um roteiro semiestruturado aplicado a 28 profissionais do SAMU, sendo 19 técnicos de enfermagem, seis enfermeiros e três médicos. Obedeceu-se aos seguintes critérios de inclusão: ser trabalhador da equipe de saúde e atuar no setor há mais de seis meses. Foram excluídos os trabalhadores que estavam afastados ou em licença durante o período da coleta de dados. Os participantes foram selecionados levando em consideração os sujeitos sociais que detinham as experiências que os pesquisadores pretendiam conhecer e o encerramento das entrevistas e das observações obedeceu ao critério de saturação teórica dos dados.

A coleta dos dados ocorreu no período de abril a junho de 2018 e a análise do material resultante das observações e da transcrição das entrevistas deu-se em conjunto, empregando-se a técnica de análise de conteúdo temática nas etapas de pré-análise, exploração do material e tratamento dos resultados ${ }^{11}$.

Respeitou-se o anonimato, nomeando os participantes com as letras "TE" para técnicos de enfermagem, "E" para enfermeiros e " $M$ " para médicos, seguido de um número que indica a ordem das entrevistas. Para as observações, utilizou-se a letra "O", seguida de um número que indica a ordem em que foram observadas. $O$ estudo obedeceu aos aspectos éticos sobre pesquisa com seres humanos ${ }^{12}$ e foi aprovado pelo Comitê de Ética em Pesquisa da Universidade Federal do Rio Grande do Sul (CAEE 82452318.3.0000.5347) e da Secretaria Municipal de Saúde de Porto Alegre (CAEE 82452318.3.3001.5338).

\section{RESULTADOS}

A maioria dos participantes era do sexo feminino (53,57\%) e a faixa etária esteve compreendida entre 31 a 60 anos, com idade média de 41,57 anos. Sobre o tempo de atuação no SAMU, a média foi de 16,32 anos, prevalecendo o 
turno de trabalho diurno (64,29\%). Treze possuíam pós-graduação $(46,43 \%)$, sendo que $8(53,57 \%)$ eram na área de urgência e emergência.

A partir das falas dos participantes e das observações não-participantes foram compostas duas categorias que permitiram compreender os conflitos na transferência do cuidado entre as equipes do atendimento pré-hospitalar e hospitalar, são elas: (1) a superlotação dos hospitais e a pouca receptividade da equipe; (2) A regulação e as (in)definições de fluxos na rede.

\section{A superlotação dos hospitais e a pouca receptividade da equipe}

A interação entre as equipes envolvidas na transferência do cuidado pré-hospitalar tem sido, na opinião dos profissionais, comprometida pelos impasses decorrentes da superlotação dos serviços. Os trechos a seguir elucidam essa questão:

[...] a gente tem uma má receptividade em quase todos os locais, acho que devido à demanda, os serviços estão sobrecarregados, então chega o SAMU, tu já não és bem visto ali, parece que tu estás trazendo serviço para os locais, sendo que tu também foi atender uma demanda, tentando dar continuidade para aquele atendimento. [...] Acontece de nem deixarem abrir a porta da ambulância para descer o paciente, mas eu digo que não tem outro local, vai ter que ser aqui mesmo, tem que ser na marra, daí aquilo gera um atrito, um jogo de empurra (TE-17).

[...] com a superlotação das emergências nós não somos bem recebidos, é como se o SAMU inventasse pacientes, tirasse pacientes do bolso (ME-01).

E possível identificar interações potencialmente capazes de comprometer a transferência do cuidado, as quais são manifestas por meio de conflitos geradores de indisposições entre as equipes:

[...] acho que é muito chato quando tu chegas num serviço e tu estás com o teu paciente ali na frente e eles falam: oh de novo, mais um?! Por que tu trouxeste agora?! tu não falaste que não vinha mais?!. Não estão nem olhando para o paciente, se está dispneico, cianótico, eles querem olhar para ti e dizer: mais um?! (TE-05).

[...] ]as pessoas ficam bem exaltadas, elas ficam mais ásperas com as equipes porque elas acham que estamos levando paciente por conta própria (TE-07).

[...] o modo como nos recebem, com piadas, questionamentos, tentando desmentir aquilo que tu estás passando (TE-17).

[...] somos mal recebidos [...] eles passam por ti e fazem de conta que nem te enxergam, como se tu não existisses, já fiquei meia hora esperando para alguém me atender (TE-14).

[...] te técnico de enfermagem fala [durante o trajeto da ambulância] que tem muita dificuldade com as portas de entrada, pois "batem pé" para aceitarem paciente (O-1).

Segundo os participantes do estudo, a retenção das macas de transporte do SAMU tem sido estratégia utilizada pelos profissionais do intra-hospitalar para conter as transferências e até para "punir" o SAMU:

[...] prendem a nossa maca sem necessidade [...] para dar um castigo (TE-08).

[...]tem dias que é guerra mesmo, tem muitos locais que tem profissionais que interpretam assim: eu vou segurar tua maca, porque se eu fizer isso tu não vais trazer outro paciente para mim (TE-11).

[...] ]eles reterem nossas macas, e isso prejudica o próximo atendimento, às vezes eu fico quatro, cinco horas com uma maca retida (TE-13).

Constata-se assim que, além de ser causa dos conflitos, a superlotação dos serviços pode estar sendo usada como justificativa para frear o trabalho do SAMU, acarretando a sensação de injustiça pelo controle exercido.

\section{A regulação e as (in)definições de fluxos na rede}

A regulação dos casos atendidos pelo SAMU e dos seus encaminhamentos enfrenta inúmeros desafios relacionados à comunicação acerca dos atendimentos e da pactuação prévia sobre o destino dos pacientes transportados pelo SAMU para os hospitais, os quais por vezes são geradores de impasses entre as equipes na transferência do cuidado. As falas a seguir exemplificam essa constatação:

[...] ocorre também que a regulação não passa a real situação do paciente e quando chegamos com o mesmo eles constatam um problema bem diferente e geralmente mais grave do que a regulação passou para o hospital. Isso gera muito atrito. Por falta de informações fidedignas (TE-01).

[...] a enfermeira não queria aceitar o paciente [...] ela diz para levar para o hospital B. O técnico diz que a regulação mandou para o hospital $A$. A enfermeira diz que a regulação estava ciente da restrição (O-2).

[...] tem tantos desafios, o primeiro é eles aceitarem o paciente, que muitas vezes é regulado pelo médico para tal local e chegam lá e barram, não aceitam (TE-13). 
[...]lsso seria muito facilitado se os médicos reguladores fizessem contato com o nosso destino, para facilitar a comunicação e o acolhimento da equipe e principalmente do nosso paciente (TE-15).

Acrescido a isso, definem-se fluxos para manobrar os direcionamentos, a fim de buscar soluções para o encaminhamento dos pacientes, mas que não ficam eximidos de discordâncias entre as equipes:

[...] O SAMU entra pela sala amarela, justificando que paciente teve perda de consciência. Residente médica questiona a classificação feita pelo SAMU. O técnico me diz que isso é um problema, pois o SAMU que "tria" a sala que o paciente vai entrar no hospital A e sempre eles discordam [...] a enfermeira diz que a regulação estava ciente da restrição do hospital A. Técnico do SAMU liga para regulação e a mesma diz que é para tentar manter no hospital A (O-2).

[...]A resposta do local para onde vai às vezes demora. Se não for paciente grave, a gente mesmo leva e regula (TE-04).

Os entraves relacionados à regulação dos casos revelam a falta de articulação entre os serviços na rede, o que também ocorre com outros fluxos operacionais, ocasionando situações em que os vínculos profissionais são afetados e se sobrepõem à organização dos serviços:

[...] às vezes é um paciente vaga-zero, vai tipo "goela abaixo" então às vezes tu não já sabe como vai ser recebido (E-02).

[...] eu fiz o boletim de ocorrência contra o médico. O protocolo do SAMU: paciente convulsiona, tem que entrar em maca e ficar em maca. Esse já tinha convulsionado três vezes, não tinha tomado a medicação, ele iria convulsionar de novo. Vai colocar esse paciente numa cadeira, deixar ele lá na frente? Ele vai cair e além da convulsão vai ter um trauma e quem vai se responsabilizar por isso? [...] Ordens absurdas eu não cumpro, foi aí que deu a confusão toda comigo. (TE-10).

\section{DISCUSSÃO}

A transferência do cuidado é um elemento chave para a continuidade dos cuidados assistenciais, no entanto, se observam altas taxas de degradação de informações clínicas importantes que acabam não sendo compartilhadas. Diante disso, estudos apontam que uma comunicação eficaz depende da objetividade na transmissão das informações e sistematização dos registros ${ }^{13-14}$ e que é preciso investir em planos de melhorias para os processos de comunicação ${ }^{15}$.

A complexidade das transferências está relacionada a elementos de comunicação, informação, organização, infraestrutura, profissionalismo, responsabilidade, conscientização da equipe e cultura. Além desses, a falta de acolhimento adequado e a falta de tempo corroboram para tal ${ }^{5,16}$. A comunicação verbal e escrita escassa durante as transferências é descrita como um dos principais componentes que prejudicam a realização de uma transferência efetiva e de qualidade ${ }^{17}$.

Os problemas estruturais da rede de atenção à saúde, principalmente relacionados à superlotação dos hospitais e à falta de estrutura física, de equipamentos, de profissionais e da disponibilidade de leitos, contribuem para a ocorrência de conflitos na transferência do paciente entre os serviços. Essa queixa é diária nos serviços de urgência e emergência ${ }^{18}$. Essas relações de trabalho fragilizadas tensionam as equipes gerando inúmeras negociações e definições de limites territoriais, que acabam promovendo a falta de um atendimento adequado e efetivo, sendo que a discordância entre os profissionais pode vir a comprometer a continuidade do cuidado ${ }^{19}$.

Paramédicos relatam que vários profissionais interrogam informações do paciente, mas não repassam as informações uns para os outros. Além disso, enfermeiras do departamento de emergência realizavam a triagem do paciente sobre as macas do serviço pré-hospitalar e, por vezes, eram interrompidas por outros profissionais, aumentando o tempo de espera dos paramédicos para transferir o paciente, bem como ocupando uma maca necessária para outros atendimentos. Uma das principais causas dessa demora está atribuída à superlotação dos departamentos de emergência, acarretando fadiga e sobrecarga de trabalho para ambos profissionais p $^{7,20,21}$.

As condições de trabalho dos profissionais nos serviços móveis de urgência indicam que os desafios aumentaram juntamente com o aumento da demanda populacional. Esses profissionais estão sobrecarregados e, nesse contexto, se estabelece precariedade nas relações devido à intensificação do trabalho ${ }^{22}$. Diante disso, os resultados do presente estudo mostraram que a interação entre o SAMU e os outros componentes da RAU nem sempre favorece uma linha de cuidado segura, pois a relação estabelecida com as equipes dos hospitais é descrita de maneira conflituosa.

As interações entre as equipes pré e intra-hospitalar, tão fundamentais à continuidade da assistência, encontrase permeada pela ocupação dos serviços e disponibilidade de leitos. A oferta da rede tem se mostrado incompatível com a demanda atual e os hospitais de grande porte, que são referências para o SAMU, frequentemente estão superlotados. Diante disso, a prerrogativa da vaga zero, que impede que o hospital de referência recuse atendimento alegando falta de leitos disponíveis. Mesmo que esse fluxo esteja regulado, essa prática realizada pelo SAMU gera certa 
resistência nos profissionais do ambiente hospitalar, que pode ser observada em pequenas atitudes, como a culpabilização do SAMU pelo aumento da carga de trabalho, originando relações conflituosas ${ }^{18,23}$.

A fragmentação dos serviços de saúde, bem como o não funcionamento adequado dos fluxos e referências, tem corroborado para a maximização dos conflitos entre as equipes do atendimento pré-hospitalar e intra-hospitalar. Segundo estudo australiano, há relatos de que os profissionais do departamento de emergência muitas vezes atêm-se a realizar outras tarefas e não recebem os pacientes transportados pelas ambulâncias, e muitas vezes, fazem 'vista grossa' e deixam os paramédicos e macas por mais tempo ocupados com esses pacientes ${ }^{7}$.

Esses resultados vão ao encontro dos resultados da presente pesquisa e de outro estudo brasileiro que revelou que os profissionais do SAMU relatam resistência em abrir portas, retenção de macas rígidas com o intuito de que o serviço pré-hospitalar não transporte mais nenhum paciente, bem como em situações mais impactantes, esses profissionais pronunciam palavras agressivas e xingamentos ${ }^{24}$.

Relações baseadas em desconfianças e mal-entendidos durante a transferência do cuidado ${ }^{20}$ comprometem a continuidade do cuidado seguro. A insatisfação dos enfermeiros de um estudo holandês foi às custas do tempo de espera e da falta de profissional médico durante a transferência do cuidado ${ }^{25}$. Ademais, estudo realizado com profissionais do pré-hospitalar na Escócia, cita que as interrupções, a variabilidade, a falta de coordenação e de um processo estruturado, retratam barreiras que impedem que a transferência seja realizada de maneira efetiva ${ }^{17}$.

O comportamento do profissional durante a transferência implica na qualidade da comunicação interprofissional. A realização de multitarefas ou de tarefas não urgentes, contribuíram para distrações e interrupções durante a transferência ${ }^{26,27}$. As interrupções são vistas pelos profissionais como uma falta de respeito, assim como a dificuldade para transferir a responsabilidade do paciente entre os profissionais foi um fator que contribuiu para a falha da comunicação ${ }^{26}$. Destaca-se que boas relações interprofissionais são imprescindíveis pra uma comunicação eficaz durante as transferências do cuidado.

Além da demanda não pertinente que compromete a organização dos fluxos do SAMU, a fragilidade da regulação também é atribuída à capacitação escassa do médico regulador ${ }^{23}$, sendo a regulação dos atendimentos uma das causas implicadas nos conflitos entre as equipes na transferência do cuidado pré-hospitalar. Acerca disso, percebe-se que, por mais que o aspecto normativo da regulação esteja bem descrito nas portarias, pode ocorrer concepções divergentes de urgência entre médico e paciente. Uma das principais dificuldades está a falta de capacitação dos médicos reguladores e a outra é a formação centrada nos aspectos técnicos de atendimento, insuficiente para regular sob uma compreensão ampliada de urgência, capacitação pontual, ausência de política de educação permanente, a baixa adesão dos mesmos a essa prática, bem como a alta rotatividade desses profissionais ${ }^{1,23}$.

Ainda sobre a regulação, estudo evidenciou desempenho insatisfatório, principalmente relacionado aos indicadores de informações atualizadas e autonomia. A comunicação com os serviços hospitalares não tem sido suficientemente eficaz, de modo que a impossibilidade de receber novos pacientes em decorrência da superlotação, da ausência de profissionais ou da falta de equipamentos não tem sido informações disponíveis para os reguladores ${ }^{18}$.

A atuação mais efetiva da regulação permite que o departamento de emergência se organize internamente, quanto ao espaço físico e a distribuição de tarefas da equipe. As normas federais instituem que a rede de saúde deve disponibilizar para a regulação do SAMU a situação da disponibilidade do serviço em receber pacientes, bem como a disponibilidade de equipamentos. Porém, esses acordos entre os gestores não se aplicam na prática, pois o acesso aos leitos muitas vezes depende de relações pessoais por parte dos médicos, sendo que alguns não se sentem obrigados a cumprir os acordos sobre referências na rede e, assim, os conflitos interprofissionais ocorrem devido ao fato de que nem sempre os profissionais se sentem comprometidos em cumprir com os acordos estabelecidos entre os gestores ${ }^{23}$.

Essas situações estabelecidas durante a transferência do cuidado interferem diretamente na prestação de atendimento adequado e qualificado aos pacientes, uma vez que ocasionam conflitos prejudiciais à comunicação. A literatura internacional também aponta que os profissionais que atuam em ambulâncias se sentem insatisfeitos com o tempo de espera ao passo que a satisfação dos profissionais dos departamentos de emergência é afetada pelo uso de instrumentos de transferência e pela integridade das informações transferidas ${ }^{25}$. O relacionamento entre os profissionais de saúde envolvidos na transferência do cuidado podem ser fragilizados pela lacuna existente entre o planejamento e a organização do trabalho, sendo necessários investimentos no que tange ao gerenciamento de conflitos e avanços na comunicação entre os profissionais ${ }^{28}$.

\section{Limitação do estudo}

Com relação à limitação do estudo, destaca-se a perspectiva dada aos profissionais do ambiente pré-hospitalar. Todavia, tais limitações não invalidam os resultados da pesquisa, mas indicam a necessidade da realização de novos estudos acerca da temática. 


\section{CONSIDERAÇÕES FINAIS}

A superlotação dos serviços de saúde, em especial das unidades de emergência, foi vista como responsável por configurar relações interpessoais conflituosas entre as equipes durante a transferência do cuidado do atendimento pré e intra-hospitalar, uma vez que traz sobrecarga aos profissionais e ocasionam um 'jogo de empurra-empurra' entre os serviços para ver quem se responsabiliza pelo paciente. Diante dessas circunstâncias, os profissionais do SAMU se sentem culpabilizados através de comportamentos de resistência em receber os pacientes, bem como punidos por meio da retenção das macas da ambulância nos serviços intra-hospitalares.

Ainda, a fragilidade da regulação e a (in)definição dos fluxos na rede também configuram fatores que ocasionam conflitos entre as equipes, pois há lacunas nas pactuações sobre o destino dos pacientes, recaindo aos profissionais o desafio de dar sequência ao atendimento.

Diante desses achados, concluiu-se que o excesso de demandas e a baixa articulação dos serviços na rede causam conflitos entre as equipes de saúde na transferência do cuidado pré-hospitalar, uma vez que os conflitos vivenciados durante a transferência afetam diretamente a segurança dos pacientes por incidir sobre as relações interpessoais e, por conseguinte sobre os processos de comunicação entre os profissionais das equipes envolvidas. Diante das causas identificadas, sugerem-se investimentos na pactuação dos processos e fluxos da rede e sistematização das comunicações entre as equipes envolvidas na transferência do cuidado pré-hospitalares com vistas a melhorar a qualidade dos encontros das equipes, tão imprescindíveis para a continuidade do cuidado e segurança dos pacientes.

\section{REFERÊNCIAS}

1. O'Dwyer G, Konder MT, Reciputti LP, Macedo C, Lopes MGM. Implementation of the Mobile Emergency Medical Service in Brazil: action strategies and structural dimension. Cad. Saúde Pública [Internet]. 2017 [cited 2019 Nov 11]; 33(7):1-14. DOI: http://dx.doi.org/10.1590/0102-311x00043716

2. Coleman EA, Boult C. Improving the quality of transitional care for persons with complex care needs. Journal of the American Geriatrics Society [Internet]. 2003; [cited 2019 Nov 18]; 51(4):556-557. DOI: https://doi.org/10.1046/j.1532-5415.2003.51186.x

3. Yong G1, Dent AW, Weiland TJ. Handover from paramedics: observations and emergency department clinician perceptions. Emerg Med Australas [Internet]. 2008; [cited 2019 Nov 11]; 20(2):149-55. DOI: https://doi.org/10.1111/j.17426723.2007.01035.x

4. Merten H, Galen LSV, Wagner C. Safe handover. BMJ [Internet]. 2017; [cited 2019 Nov 11]; 359. DOI: https://doi.org/10.1136/bmj.j4328

5. Panchal AR, et al. The impact of professionalism on transfer of care to the emergency department. The Journal of Emergency Medicine [Internet]. 2015 [cited 2019 Nov 15]; 49(1):18-25. DOI: https://doi.org/10.1016/j.jemermed.2014.12.062

6. Queensland Ambulance Service. Clinical Practice Procedures: Other/Clinical handover. Clinical Quality and Patient Safety Unit [Internet]. 2015 [cited 2019 Nov 11]; Available from: https://www.ambulance.qld.gov.au/docs/clinical/cpp/CPP_Clinical\%20handover.pdf

7. Bost MN, Crilly J, Patterson E, Chaboyer W. Clinical handover of patients arriving by ambulance to a hospital emergency departament: a qualitative study. Intern Emerg Nurs [Internet]. 2012 [cited 2019 Nov 11]; 20(3):133-41. DOI: https://doi.org/10.1016/j.ienj.2011.10.002

8. The Joint Commission. Joint Commission International Accreditation Standards for Hospitals [Internet]. 2014 [cited 2019 Jan 18]; Available from: http://www.jointcommissioninternational.org/assets/1/14/JClH14_Sample_Pages.pdf

9. Ministério da Saúde (BR). Assistência Segura: Uma Reflexão Teórica Aplicada à Prática [Internet]. Brasília (DF); 2017 [cited 2019 Feb 02]. Available from: http://portal.anvisa.gov.br/documents/33852/3507912/Caderno+1++Assist\%C3\%AAncia+Segura++Uma+Reflex\%C3\%A3o+Te\%C3\%B3rica+Aplicada+\%C3\%A0+Pr\%C3\%A1tica/97881798-cea0-49749d9b-077528ea1573

10. Lima DP, Leite MTS, Caldeira AP. Health Care Networks: the perception of physicians working in emergency services. Saúde debate [Internet]; 2015 [cited 2019 Jan 14], 39(104):65-75. DOI: https://doi.org/10.1590/0103-110420151040348

11. Minayo MCS. O desafio do conhecimento: pesquisa qualitativa em saúde. 13. ed. São Paulo: Hucitec, 2013.

12. Ministério da Saúde. Resolução no 466, de 12 de dezembro de 2012. Brasília: Ministério da Saúde, 2012. Dispõe sobre as diretrizes e normas regulamentadoras de pesquisas envolvendo seres humanos. Diário Oficial da União [Internet], Brasília (DF) 12 de dezembro de 2012 [cited 2019 Jan 14]. Available from: http://bvsms.saude.gov.br/bvs/saudelegis/cns/2013/res0466_12_12_2012.html

13. Silva MF, Anders JC, Rocha PK, Souza AIJ, Burciaga VB. Communication in nursing shift handover: pediatric patient safety. Texto contexto - enferm [Internet]. 2016 [cited 2019 Nov 12]; 25(3):1-9. DOI: https://doi.org/10.1590/0104-07072016003600015

14. Olino L, Gonçalves AC, Strada, JKR, Vieira LB, Machado MLP, Molina L, Cogo ALP. Effective communication for patient safety: transfer note and Modified Early Warning Score. Rev. Gaúcha Enferm [Internet]. 2019 [cited 2019 Oct 13]; 40(esp):1-9. DOI: https://doi.org/10.1590/1983-1447.2019.20180341

15. Hemesath MP, Kovalski AV, Echer IC, Lucena AF, Rosa NG. Effective communication on temporary transfers of inpatient care. Rev. Gaúcha Enferm. [Internet]. 2019 [cited 2019 Nov 11]; 40(spe):e20180325. DOI: https://doi.org/10.1590/19831447.2019.20180325 
16. Quiles AS, Ramón MDPH, Sanchis RJ, Aracil NG, Encina MEC, Galvañ JP. Handover of patients from prehospital emergency services to emergency departments: a qualitative analysis Based on Experiences of Nurses. J Nurs Care Qual [Internet]. 2019 [cited 2019 Oct 14]; 34(2):169-74. DOI: https://dx.doi.org/10.1097\%2FNCQ.0000000000000351

17. Fitzpatrick, McKenna M, Duncan EAS, Laird C, Lyon R, Corfield A. Critcomms: a national cross-sectional questionnaire based study to investigate prehospital handover practices between ambulance clinicians and specialist prehospital teams in Scotland. Scand J Trauma Resusc Emerg Med [Internet]. 2018 [cited 2019 Nov 11]; 26(45):45. DOI: https://dx.doi.org/10.1186\%2Fs13049018-0512-3

18. Ortiga AMB, Lacerda JT, Natal S, Calvo MCM. Evaluation of the Mobile Emergency Care Service in Santa Catarina State, Brazil. Cad Saúde Pública [Internet]. 2016 [cited 2019 Oct 21]; 32(12):1-13. DOI: http://dx.doi.org/10.1590/0102-311x00176714

19. Torres SFS, Belisario SA, Melo EM. The emergency network in the northern macro-region of Minas Gerais, Brazil: a case study. Saúde Soc. [Internet]. 2015 [cited 2019 Nov 11]; 24(1): 361-373. DOI: http://dx.doi.org/10.1590/S0104-12902015000100028

20. Reay G, Norris JM, Hayden KA, Abraham J, Yokom K, Nowell L, Lazarenko GC, Lang ES. Transition in care from paramedics to emergency department nurses: a systematic review protocol. Syst Rev [Internet]. 2017 [cited 2019 Nov 11]; 6(1):260. DOI: https://doi.org/10.1186/s13643-017-0651-z

21. Reay G, Rankin JA, Then KL. Momentary fitting in a fluid environment: A grounded theory of triage nurse decision making. Int Emerg Nurs [Internet]. 2016 [cited 2019 Nov 11]; 26:8-13. DOI: https://doi.org/10.1016/j.ienj.2015.09.006

22. Dal Pai D, Lima MADS, Abreu KP, Zuxatti PB, Lautert L. Teams and working conditions in mobile pre-hospital care services: an integrative review. Rev. Eletr. Enferm. [Internet]. 2015 [cited 2019 Nov 11]; 17(4):1-12. Available from: https://www.google.com.br/url?sa=t\&rct=j\&q=\&esrc=s\&source=web\&cd=\&cad=rja\&uact=8\&ved=2ahUKEwjJs_f9vfqAhX1HrkGHYUOD78QFjABegQIARAB\&url=https\%3A\%2F\%2Fwww.revistas.ufg.br\%2Ffen\%2Farticle\%2Fdownload\%2F31522 \%2F20661\%2F\&usg=AOvVaw1vvAWqKu7guTD2umdw_Ko4

23. O'Dwyer G, Machado CV, Alves RP, Salvador FG. Mobile prehospital emergency care: an analysis of implementation in the State of Rio de Janeiro, Brazil. Ciência \& Saúde Coletiva [Internet]. 2016 [cited 2019 Nov 11]; 21(7):2189-200. DOI: https://doi.org/10.1590/1413-81232015217.15902014

24. Mello DB. Dispositivos de proteção utilizados por profissionais de atendimento pré-hospitalar móvel frente à violência no trabalho [Internet, dissertação]. Porto Alegre: Escola de Enfermagem, Universidade Federal do Rio Grande do Sul, Porto Alegre; 2015 [cited 2019 Nov 11]. Available from: https://lume.ufrgs.br/bitstream/handle/10183/135470/000987783.pdf?sequence=1\&isAllowed

25. Hovenkamp ST, Olgers TJ, Wortel RR, Noltes ME, Dercksen B, Maaten JC. The satisfaction regarding handovers between ambulance and emergency department nurses: an observational study. Scand J Trauma Resusc Emerg Med [Internet]. 2018 [cited 2019 Nov 11]. DOI: https://dx.doi.org/10.1186\%2Fs13049-018-0545-7

26. Redley B, Botti M, Wood B, Bucknall T. Interprofessional communication supporting clinical handover in emergency departments: An observation study. Australas Emerg Nurs J [Internet]. 2017 [cited 2019 Nov 11].]; 20(3):122-30. DOI: http://dx.doi.org/10.1016/j.aenj.2017.05.003

27. Giske T, Melås SN, Einarsen KA. The art of oral handovers: A participant observational study by undergraduate students in a hospital setting. J Clin Nurs [Internet]. 2018 [cited 2019 Nov 11]; 27(5-6):767-75. DOI: https://doi.org/10.1111/jocn.14177

28. Worm FA, Pinto MA, Schiavenato D, Ascari RA, Trindade L, Silva OM. Risk of disease of nursing professionals at work in emergency mobile service. RevCuid [Internet]. 2016 [cited 2019 Nov 11]; 7(2):1288-96. DOI:

https://doi.org/10.15649/cuidarte.v7i2.329 